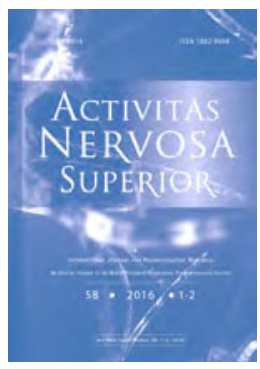

\title{
ASSOCIATION BETWEEN DYSTHYMIC DISORDER AND DISABILITY, WITH RELIGIOSITY AS MODERATOR
}

\author{
Ravinder S. Sandhu*1, Samiran Ghosh², Timothy Dellenbaugh \\ ${ }^{1}$ Department of Psychiatry, Center for Behavioral Medicine, University of Missouri Kansas City, USA \\ 2Department of Family Medicine E Public Health Sciences and Center for Urban Response to Environmental \\ Stressors, University of Missouri Kansas City, USA
}

\begin{abstract}
There has been much research on Major Depressive Disorder (MDD) in terms of functional impairment associated with the disorder. On the other hand, Dysthymic Disorder (DD) has not received much attention in the literature in relation to the functional disability associated with the disorder. Similarly, the moderator effect of religiosity has been studied in many physical and mental health disorders, but has not been explored in DD. The aims of the present study were to explore the association between DD and functional disability, and to study the moderator effect of religiosity on the association. The data for the study was obtained from consortium of Psychiatric Epidemiologic Survey (CPES). The survey collected data on prevalence of mental health problems and the sociocultural determinants, in the national population. The present study utilized the data on DD and factors such as race, gender, marital status, employment status, and religiosity, in order to explore association between DD and disability and the effect of religiosity on the association. Results show that the subjects who have endorsed DD are 1.092 times more likely to have functional disability than those who have not endorsed DD. The association is statistically significant with the $95 \%$ CI for the Odds Ratio (OR) as $1.072-1.113$. The religiosity has not shown a significant effect on the association between DD and functional disability. In conclusion there is statistically significant association between DD and the functional disability. Although the cross-sectional study design makes it difficult to determine the causal relationship, the results may signify that DD could potentially cause significant functional impairment. Further longitudinal studies are needed to probe the association between DD and disability.
\end{abstract}

Key words: Major Depressive Disorder (MDD); Functional impairment; Religiosity; Socio-cultural factors

\section{INTRODUCTION}

Dysthymic Disorder (DD) is a milder form of Major Depressive Disorder (MDD), with the presence of mild depressive symptoms for 2 years. In DSM-5, the diagnosis of DD and chronic depression are combined as Persistent Depressive Disorder. The undertaking of combining the two diagnoses reflects the equal extent of distress and impaired life quality of people suffering from the disorders, MDD (chronic) and DD. According to American Psychiatric Association, there is 6\% life time prevalence of Dysthymic disorder (with or without superimposed Major Depressive Disorder), and 3\% point prevalence of the disorder (Devanand, 2014). The little

*Correspondence to: Ravinder S. Sandhu; e-mail: rssandhu77@yahoo.com

Received November 16, 2015; accepted May 15, 2016; Act Nerv Super 58(1-2), 13-19; ISSN-1802-9698 
research done on DD has shown that disability resulting from the disorder is even greater than that from MDD (Hellerstein et al., 2010). This study has shown the adverse impact of the DD on occupational and psychosocial functioning of general population. A study conducted with 97 early-onset DD outpatients and 45 MDD episode outpatients (Klein et al., 2000a; Klein et al. 2006b) has demonstrated the association of DD with significant impairment in psychosocial functioning, along with a high rate of suicide attempts and psychiatric hospitalizations. The Medical Outcomes Study assessed 11,242 patients receiving treatment from Health Maintenance Organizations (HMOs) and different specialty practices, including 76 MDD patients and 48 DD patients. The study group found that DD was associated with impairment in role functioning, general health, psychosocial functioning and physical functioning (Wells et al., 1992). Also, over the last 2 decades, a substantial body of literature evaluating the associations between parameters of religiosity and depression has been generated. One study (Koenig, McCullough \& Larson, 2001) demonstrated lower odds of having depression in adults who are religious to a greater extent. Religiosity not only seems to have protective role against developing depression, but also it could potentially reduce the disability resulting from depression. A study conducted in an elderly population (Santos et al., 2013) has shown that religious beliefs and traditions assists elderly to explain and address the suffering experienced by them as a result of existing or imminent functional disability.

The few studies conducted on probing the association between the DD and disabilities have used relatively small sample sizes. It has not been studied whether the potential association between DD and disability holds true for nationally representative population. Past studies have shown negative associations between religiosity and depression. If a positive association exists between DD and disability, what traits may affect this association? It will be helpful to know whether sociocultural factors such as belief in religion may influence the association between DD and disability.

The present study is designed to examine the association between DD and functional disability in a national population of the United States. The large sample of the study is recruited from all the states of the U.S.A. Sociocultural factors such as race, gender, socioeconomic status, and age have been included in the statistical analysis to better discern the dynamics of association between DD and disability. Furthermore, the study aims to probe the effect of belief in religion on the association between DD and disability. The previous studies suggest that religion has a protective effect against development of depression. This study examines whether religiosity also decreases the subjective report of disability from DD. The study is conducted to test two hypotheses, first, there is direct association between DD and disability, and second, belief in religion moderates the subjective disability from DD. The expected outcomes of the study are findings of direct associations between DD and functional disability, and an inverse association between the degree of religiousness and the subjective functional disability from DD.

\section{METHODS}

2.1. Source of data: The Consortium in Psychiatric Epidemiology Survey (CPES) data is used in this study. The CPES conducted three national surveys, namely National Comorbidity Survey- Revised (NCS-R), National Survey of American Life (NSAL), and National Latino and Asian American Study (NLAAS). The objective of the CPES project was to explore the prevalence of mental health disorders in the American population, and to demonstrate the role of socioeconomic and cultural factors in mental health problems' causation and management, especially in minority ethnic populations. The data used for the present study consisted of 20013 subjects.

2.2. Study Variables: DD was the outcome variable of interest. In DSM IV, it is defined as presence of mild depressive symptoms for at least 2 years. The disorder was measured as "endorsed" or "not endorsed" based on response to survey questions. Also, functional 
disability was measured as "endorsed" or "not endorsed" depending on whether the subject had performed less than their full potential at work for any number of days in the past 30 days. The other independent variables included in the study were race, gender, marital status, employment, and religiosity. Marital status also was measured as a categorical variable"married/cohabiting", "divorced/separated/widowed", and "never married". The employment status was categorized as "employed", "unemployed", and "out of labor force". Religiosity has been defined variously, one of the common definitions of religiosity is an organized system of beliefs, practices, and rituals adapted to gain closeness to the sacred God (Moreira-Almeida, Neto, Koenig, 2006). Religiosity in the original study was measured as a categorical variable coded as "very religious", "fairly religious", "not too religious", and "not religious at all". The subjects were assigned a category based on the reported intensity of belief in religion. For our purpose of evaluating moderation analysis we have combined the first two categories and last two categories of religiosity to make it a binary variable. This has been done as the interaction term between religiosity and disability which quantifies the moderation effect, is much easier to interpret when at least one of the covariate is binary in nature.

2.3. Data analysis: A sample of 20013 subjects was initially used to conduct statistical analysis. The study sample was recruited from all the states of the U.S.A. representing its national population. There were about $45 \%$ males and $55 \%$ females. The sample consisted of 9\% Caucasians, 3\% African Americans, 48\% Hispanics, and 39\% Asians. A binary logistic regression model was used to examine the association between functional disability and DD. The model used the stepwise inclusion of the independent variables of functional disability, race, employment, gender, and marital status. A separate logistic regression model was used to analyze the moderation effect of religiosity on functional disability. The effective sample size used by regression models was reduced to 5340 subjects because of missing values on the religiosity variable. This could induce selection bias into the study because the dropping out of a large segment of subjects may represent a group based on particular criteria. A p-value of $<0.05$ was used for statistical significance of the study findings. SAS (version 9.3) software was used for the analysis.

Table1. Descriptive variables

\begin{tabular}{lll}
\hline $\begin{array}{l}\text { Variables } \\
\text { Categorical variables } \\
\text { Gender }\end{array}$ & $\mathbf{N}$ (number) & \% age \\
\hline Male & 2400 & 44.9 \\
Female (ref.) & 2940 & 55.0 \\
Race & 2101 & 39.3 \\
Asian & 2586 & 48.4 \\
Hispanics & 141 & 2.6 \\
African American & 500 & 9.3 \\
Caucasians & 12 & 0.2 \\
All others & & \\
Marital status & 3412 & 63.8 \\
Married/cohabiting & 794 & 14.8 \\
Divorced/separated/widowed & 21.2 \\
Never married (ref.) & 1134 & \\
Employment status & & 65.3 \\
Employed & 3492 & 6.5 \\
Unemployed & 351 & 28.0 \\
Not in labor force (ref.) & 1497 & 25.3 \\
Education status & & \\
0-11 years & 1355 &
\end{tabular}




\begin{tabular}{lll}
12 years & 1179 & 22.0 \\
13-15 years & 1306 & 24.4 \\
16 years and above (ref.) & 1500 & 28.0 \\
\hline DD & 175 & 3.2 \\
Endorsed & 5165 & 96.7 \\
Not endorsed & & \\
Religiosity & 1666 & 31.1 \\
Very religious & 2736 & 51.2 \\
Fairly religious & 678 & 12.6 \\
Not too religious & 238 & 4.4 \\
Not religious at all (ref.) & & \\
\hline
\end{tabular}

\begin{tabular}{lll}
\hline $\begin{array}{l}\text { Continuous variables } \\
\text { Variable }\end{array}$ & Mean & SD \\
\hline Age & 43.38 years & 16.7 \\
Functional disability & 1.37 days & 4.8 \\
\hline
\end{tabular}

\section{RESULTS}

Two separate regression models were used for the analysis of association between DD and functional disability; and to analyze the moderator effect of religiosity on the association, respectively. The both models were shown to be good-fit with Hosmer and Lemeshow goodness of fit test yielding p-values of 0.4148 and 0.3148 respectively. The high P-values for the models allow not rejecting the null effects of covariates. The regression models used a stepwise entrance of the variables. The final model for analysis of association between DD and functional disability includes five variables of gender, race, marital status, employment status, and functional disability. The model excluded the variables of education and age for their less significant contributory effect in the model. Table 2 shows that the odds of having DD among those who have functional disability are 1.092 times compared to those who did not have functional disability. The $95 \%$ CI for the OR is $1.072-1.113$. In the study sample, males with functional disability are shown to have higher odds of having DD compared to females with functional disability with an OR of 1.448 and $95 \%$ CI of $1.033-2.031$.

Among different races; Caucasians, African-Americans, and Hispanics who have functional disability are shown to have lower odds of endorsing DD compared to Asians with functional disability with an OR of $0.355,0.433$, and 0.590 (95\% CI excludes 1 for all three races) respectively. Also, married and employed subjects with disability show higher odds of endorsing DD compared to those with functional disability, and never married, and were out of labor force, respectively. The second regression model which includes all of the covariates in the first model, additionally we also include religiosity and its interaction term with functional disability. This is because moderation effects are typically measured as the interaction between factors or variables (i.e. religiosity and functional disability), where the effects of one variable depend on levels of the other variable in analysis (Fairchild \& McKinnon, 2009). Table 3 shows that religiosity did not have a moderator effect on the association between DD and functional disability for the present study sample as the interaction term between religiosity and functional disability is statistically not significant (pvalue $=0.6472$ ). Even though the moderation effect was not statistically significant for the study sample, the subjects who were very religious, fairly religious, or not too religious with functional disability have shown lower odds of endorsing DD compared to those who were not religious at all and had functional disability. 
Table 2. Results for logistic regression analysis to demonstrate the relationship between DD and functional disability.

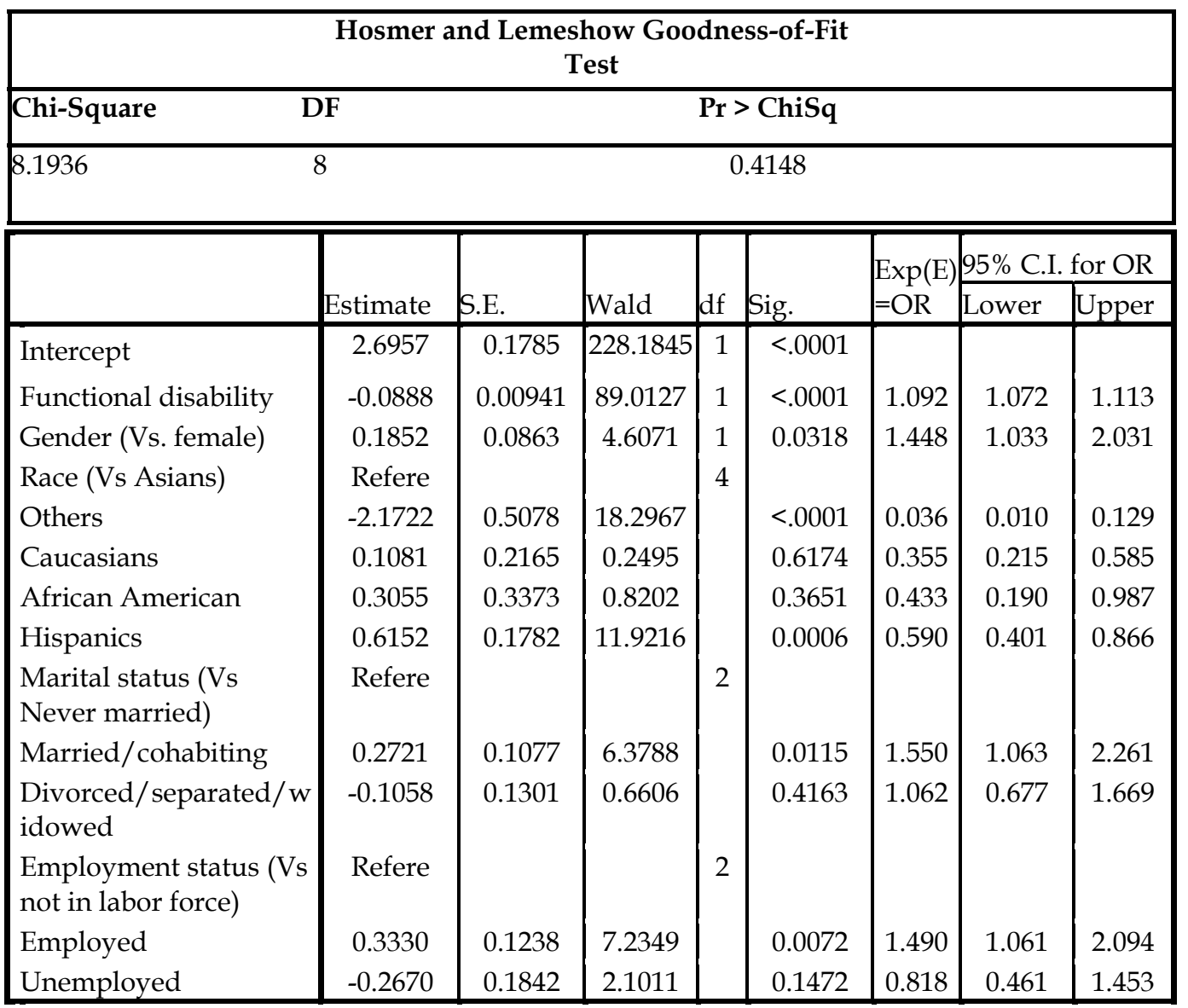

Note: Refere:- Reference Category

Table 3. Results for logistic regression analysis with religiosity used as a moderator

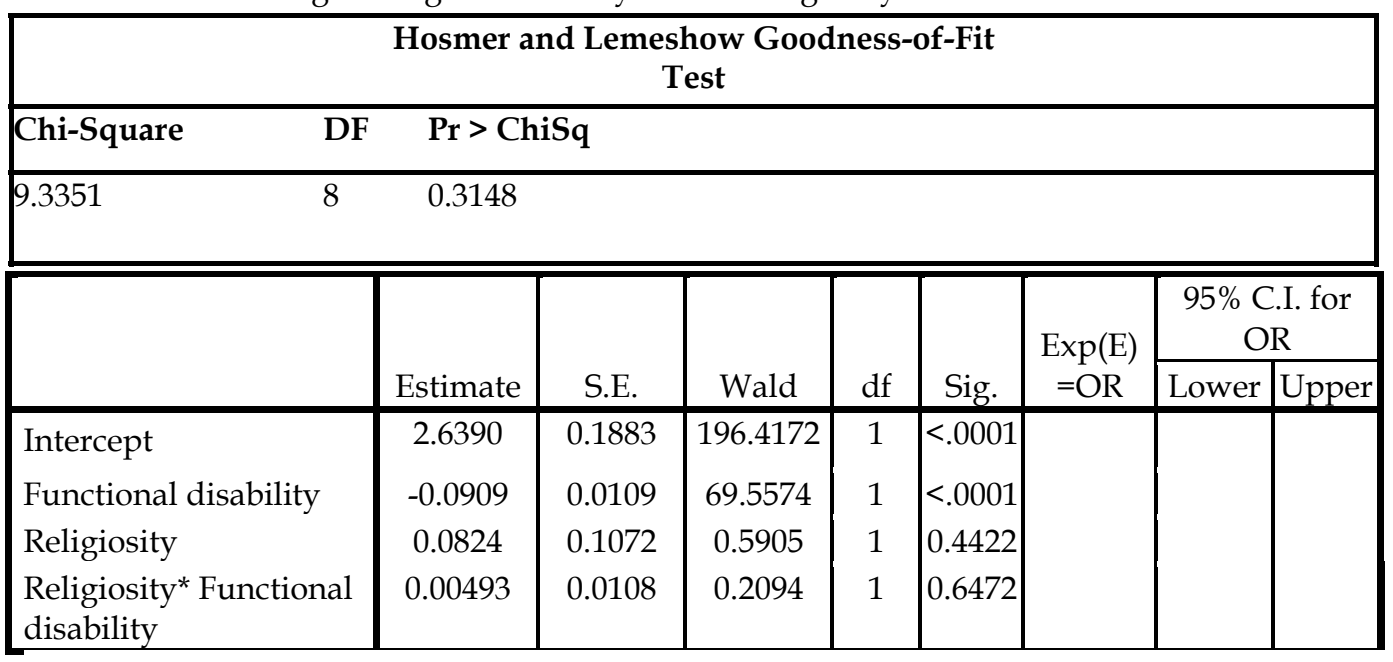




\begin{tabular}{|l|c|c|c|c|c|c|c|c|} 
Gender (Vs. female) & 0.1811 & 0.0865 & 4.3888 & 1 & 0.0362 & 1.437 & 1.024 & 2.016 \\
Race (Vs Asians) & $\begin{array}{l}\text { Refere } \\
\text { Others }\end{array}$ & & & 4 & & & & \\
Caucasians & -2.1582 & 0.5079 & 18.0587 & & $<.0001$ & 0.037 & 0.010 & 0.132 \\
African American & 0.1029 & 0.2168 & 0.2254 & & 0.635 & 0.357 & 0.217 & 0.590 \\
Hispanics & 0.3017 & 0.3383 & 0.7953 & & 0.3725 & 0.436 & 0.190 & 0.998 \\
Marital status (Vs & 0.6217 & 0.1784 & 12.1418 & & 0.0005 & 0.600 & 0.408 & 0.883 \\
Never married) & Refere & & & 2 & & & & \\
Married/cohabiting & 0.2707 & 0.1078 & 6.3041 & & 0.0120 & 1.544 & 1.057 & 2.254 \\
Divorced/separated/w & -0.1072 & 0.1303 & 0.6771 & & 0.4106 & 1.058 & 0.673 & 1.664 \\
idowed & & & & & & & & \\
Employment status (Vs & Refere & & & 2 & & & & \\
not in labor force) & & & & & & & & \\
Employed & 0.3346 & 0.1239 & 7.2904 & & 0.0069 & 1.495 & 1.064 & 2.102 \\
Unemployed & -0.2669 & 0.1841 & 2.1028 & & 0.1470 & 0.819 & 0.462 & 1.455 \\
\hline
\end{tabular}

Note: Refere:- Reference Category

\section{DISCUSSION}

The study has supported the first hypothesis that there is an association between DD and functional disability. The purpose of the present study was to emphasize the significance of Dysthymic Disorder in relation to functional disability. Numerous studies have illustrated the importance of Major Depressive Disorder (MDD) as a debilitating psychiatric disorder and the need to prevent and treat it. MDD is a serious mental health issue that in addition to causing functional, social, and occupational impairment could lead to suicide if not treated appropriately. On the other hand, the DD is also comprised of similar signs and symptoms as MDD with less intensity but for longer duration. It is apparent that as a result of longer duration of symptoms, Dysthymic Disorder could be more debilitating than Major Depressive Disorder. The present study demonstrates seriousness of DD in the form of loss of quality of life in terms of impaired functioning. The statistical analysis of the present study sample does not support the moderator effect of religiosity on the association between DD and functional disability. We would like to point out that the study data has missing values on religiosity for a large segment of subjects, which we have ignored in the current paper. Since we only performed secondary analysis of the existing data it was not clear from the original study design the reason for such a large missing values.

The primary study was conducted on a sample representing national population of the United States. Apparently, efforts were made to avoid selection bias in the study sample to make the results applicable to the general population of U.S.A. The sample was collected from all the states and consisted of people from many different races. The age range for the studied subjects was 18 through 99 years. Since the present study was conducted as a secondary analysis of existing data, there could be a concern about the data quality. The subjects took the survey in their residences. This avoids any stress or anxiety in the study participants that could otherwise arise from being surveyed in clinical or laboratory settings. As the study design was cross-sectional, there was no risk of drop out of the study participants which could be a problem in a cohort study design. On the other hand, the cross-sectional study design is unable to demonstrate "cause and effect" relationships. Dysthymic Disorder could very well lead to disability, but it could also be possible that prolonged disability causes Dysthymic Disorder. It is not possible to tell the direction of the relationship from the study design. The diagnosis of the Dysthymic Disorder in the survey was not made by a medical professional; rather it was based on questionnaire asked by a trained lay person. The study has not 
supported the moderation effect of religiosity. This could be because a large data portion could not be employed for the analysis as a result of missing values on religiosity variable. The missing data might represent a specific group of subjects in relation to religiosity beliefs, leading to systematic selection bias.

Nonetheless, the study shows a statistically significant association between Dysthymic Disorder and the functional disability. The later association should inform public health professionals to appreciate the seriousness of DD and the importance of diagnosing and treating the condition appropriately in a timely fashion to avoid the bad consequences such as functional disability. Future longitudinal studies are warranted to further explore the dynamics and direction of the association between Dysthymic Disorder and the disability. Also, future studies with more appropriate sample selection are needed to probe the moderation effect of religiosity on the association between DD and functional disability.

\section{REFERENCES}

Devanand, D.P. (2014). Dysthymic disorder in the elderly population. International Psychogeriatrics, 26, 39-48.

Fairchild, A.J. \& MacKinnon, D.P. (2009). A General Model for Testing Mediation and Moderation Effects. Prevention Science, 10, 87-99.

Hellerstein, D.J., Agosti, V., Bosi, M., \& Black, S.R. (2010). Impairment in psychosocial functioning associated with dysthymic disorder in the NESARC study. Journal of Affective Disorders, 127, 84-88.

Heeringa, S.G., Wagner, J., Torres, M., Duan, N., Adams, T., \& Berglund, P. (2004). Sampledesigns and sampling methods for the Collaborative Psychiatric Epidemiology Studies (CPES). International Journal of Methods in Psychiatric Research, 13, 221-40.

Klein, D.N., Schwartz, J.E., Rose, S., \& Leader, J.B. (2000a). Five-year course and outcome of dysthymic disorder: a prospective, naturalistic follow-up study. American Journal of Psychiatry, 157,931-939.

Klein, D.N., Shankman, S.A., \& Rose, S. (2006b). Ten-year prospective follow-up study of the naturalistic course of dysthymic disorder and double depression. American Journal of Psychiatry, 163, 872-880.

Koenig, H., McCullough, M.E., \& Larson, D.B. (2001). Handbook of religion and health. 1st ed. New York (NY): Oxford University Press.

Miller, A.S., \& Hoffmann, J.P. (1995). Risk and religion: an explanation of gender differences in religiosity. Journal for the Scientific Study of Religion, 34, 63-75.

Moreira-Almeida, A., Neto, F.L., \& Koenig, H.G. (2006). Religiousness and mental health: a review. Revista Brasileira De Psiquiatria, 28, 242-250.

Rasic, D., Kisely, S., Langille, D.B. (2011). Protective associations of importance of religion and frequency of service attendance with depression risk, suicidal behaviours andsubstance use in adolescents in Nova Scotia, Canada. Journal of Affective Disorders, 132, 389-395.

Santos, W.D., Giacomin, K.C., J.K., Pereira 1, \& Firmo, J.A. (2013). Coping with functional disability among the elderly by means of religious beliefs. Programa de Pós-Graduação em Ciências da Saúde, Laboratório de Epidemiologia e Antropologia Médica, Centro de Pesquisas René Rachou, Fiocruz. Av. Augusto de Lima 1715/610, Barro Preto. 30.190-002.

Wells, K.B., Burnam, M.A., Rogers, W.H., \& Hays, R. (1992). Course of depression for adult patients: results from the Medical Outcomes Study. Archives of General Psychiatry, 49, 788-794. 